\title{
Cognitive stories and the image of mathematics
}

\author{
Roy WAGNER
}

Received: 07/06/2017

Final version: 06/01/2018

BIBLID 0495-4548(2018)33:2p.305-323

DOI: $10.1387 /$ theoria. 17917

ABSTRACT: This paper considers two models of embodied mathematical cognition (a modular model and a dynamic model), and analyses the image of mathematics that they support.

Keywords: Mathematical cognition, Walter Freeman, embodied cognition, popular view of mathematics.

RESUMEN: Este artículo considera dos modelos de cognición matemática corporizada (un modelo modular y un modelo dinámico), y analiza la imagen de las matemáticas que apoyan.

Palabras clave: Cognición matemática, Walter Freeman, cognición corporizada, visión popular de las matemáticas.

\section{Introduction}

Paul Ernest defines the absolutist philosophies of mathematics as those for which mathematics is an "objective, absolute, certain and incorrigible body of knowledge, which rests on the firm foundations of deductive logic" (Ernest 2008, 2). These philosophies are often associated with an over-valuation of mathematics. Since mathematics is viewed as objective, absolute and a-priori, mathematical knowledge or knowledge supported by mathematics are allegedly more sound than other forms of knowledge. Such knowledge should be encouraged and promoted, even if only a handful of people understand it properly.

This point of view is reflected in a recent experiment: scholars were asked to evaluate social sciences abstracts, some of which had a sentence added to them that included a meaningless string of symbols resembling a mathematical statement. It turns out that evaluators lacking strong mathematical education tended to evaluate the abstracts with junk mathematics more positively (Erikson 2012).

Curiously, the absolutist view is sometimes also associated with an under-valuation of mathematics. According to this interpretation, mathematics is absolute and infallible because it is mechanical, inhuman and irrelevant to our dynamic and uncertain lives. The people who do mathematics are therefore strange machine-people ("nerds"), who are hard to communicate with.

Of course, as noted by Ernest, an absolutist view can be associated with positive approaches to mathematics, which are not restricted to the overvaluation mentioned above, by dissociating the absolute image of mathematical facts from a humanistic process of mathematical learning and discovery. But the view of mathematics as a process of 
human discovery is more often related to fallibilist philosophies of mathematics, which view it as "the outcome of social processes. Mathematical knowledge is understood to be fallible and eternally open to revision, both in terms of its proofs and its concepts" (Ernest 2008, 3).

Since I consider the absolutist view and its related over- and under-valuations as potentially dangerous to a fruitful engagement of the general public, intellectuals and decision makers with mathematics, I find it important to look for support for approaches that may promote fallibilism. Traditionally, cognitive science tended to be formal, abstract and computational, and therefore not much of an ally to fallibilists. But more recently, cognitive science has grown more "embodied" - that is, starting its analysis from the position of an embodied mind operating in a concrete practical-ecological niche. This view allows mathematics to evolve fallibly together with changes in our embodiment, society and environment, and, given the popularity of cognitive science, may help change the public image of mathematics (even if not the views of those committed to an absolutist first philosophy).

The purpose of this paper is, therefore, to briefly present two of the many embodied accounts of mathematical cognition, and consider the images of mathematics that they promote. While no existing cognitive theory of mathematics has, as far as I can evaluate, a sound empirical support, and while all are subject to very substantial critiques (that's why I'm referring to these accounts below as "stories" rather than "theories"), cognitive theories are readily popularized and quickly become culturally influential, regardless of their scientific evidence. Exploring the repercussions of cognitive theories on the image of mathematics will thus help us understand the cultural role that such theories can play in promoting fallibilist approaches to mathematics, and confronting its over- or undervaluation.

\section{Two Stories about mathematical cognition}

I will present here two stories about mathematical cognition. The first story derives from the theory of cognitive mathematical metaphors, associated with Lakoff and Núñez (2000), which was later enhanced by a neuro-cognitive account (Lakoff 2008). I will refer to this below as the modular story. The second story is derived from Walter J. Freeman III's general theory of cognition (Freeman 2000), whose application to mathematical cognition was only hinted at in his subsequent work (Freeman 2009, Freeman and Kozma 2009). I will refer to this as the dynamic story.

Since my purpose here is to reflect on what these stories mean for the general image of mathematics, rather than on evaluating or criticizing them, I will allow myself to be rather sketchy and vague in their presentation. The level of precision in this presentation will be restricted to what is relevant for forming a public image of mathematics, not for a proper scientific evaluation. A more detailed analysis with supportive and critical references, including my own substantial critiques, are available in (Wagner 2017, ch. 5; 2013).

These two cognitive stories (the first well known and widely cited, the second representative of more recent, tentative approaches) were chosen because I believe that they represent extreme aspects of the image of mathematics spanned by contemporary 
embodied cognitive science. The two stories can obviously be mediated in many ways, and complemented by other stories. For example, the conception of reasoning as sensory-motor simulation and the position of extended cognition are briefly mentioned in the discussion - but there are many other approaches that I simply ignore here. I allow myself this "neglect", because my point is not to provide a survey of mathematical cognition theories, but to explore possible images of mathematics emerging from cognitive studies. The two extremes cases considered here more or less bound the current range of possibilities.

\section{THE MODULAR STORY}

I refer to this story as modular, because it divides mathematical reasoning into modules or domains in the brain. Mathematical domains start at the level of more or less universal embodied actions (such as handling object collections, walking along a path). These domains are the ground on which number reasoning is constructed (adding, for example, can be viewed as combining object collections or as combining steps along a path). Then as more cognitive domains are brought into relation with the system of previous domains, more abstract mathematical domains emerge (e.g. plane geometry, algebra, calculus, etc.).

In this context, each domain is thought of as a neural circuit made of nodes, each of which represents a different mathematical situation. These nodes are internally connected by inference links - when the nodes of the premises of the inference are activated, the node representing the conclusion is activated as well. This allows new actions to follow from given premises or perceptions.

The domains are linked to each other by various kinds of mappings, the most important of which are metaphors. Metaphors are a-symmetrical links that allow to carry inferences from one domain to another. They can "ground" an abstract mathematical domain in a concrete embodied activity, or link different abstract domains (e.g. relate number-reasoning to handling discrete objects, or algebraic inferences to geometric ones).

Metaphors form when different domains are activated together frequently (because they are both relevant in a certain kind of situation, such as using numbers when handling object collections). According to Hebb's law, frequently co-activated nodes tend to form neural links (under some qualifications that we can set aside in this paper). These neural links transmit activation between the connected nodes. For example, nodes of the target domain (e.g. number addition) may be activated when the corresponding nodes of the source domain (e.g. putting together collections of objects) are activated.

In the example of the "numbers are object collections" metaphor, inferences involved in handling object collections are transferred by the metaphor to the domain of number (e.g., the fact that the same object collection can be composed in different ways is translated to the claim that the same number can be the result of different addition operations). Table 1 presents a more complex example: how the cognitive domain of the number line emerges from a metaphor linking the continuous line with a set of elements (turning the lines into the set of its points), and then blending it with numbers, forming the number line (a blend is a stronger, bi-directional kind of mapping, but the precise differences are not important for our context). 


\section{Table 1}

The metaphors and blends generating the number line, based on Lakoff and Núñez (2000, 281)

\begin{tabular}{|c|c|c|}
\hline \multicolumn{2}{|c|}{$\begin{array}{l}\text { Source Domain } \\
\text { The Space-Set Blend }\end{array}$} & \multirow{2}{*}{$\begin{array}{c}\text { Target Domain } \\
\text { Numbers }\end{array}$} \\
\hline $\begin{array}{c}\text { Naturally Continuous Space: } \\
\text { The Line }\end{array}$ & Sets & \\
\hline The line & A set & A set of numbers \\
\hline Point-locations & Elements of the set & Numbers \\
\hline Points are locations on the line & $\begin{array}{l}\text { Elements are members of the } \\
\text { set }\end{array}$ & $\begin{array}{l}\text { Individual numbers are members of } \\
\text { the set of numbers }\end{array}$ \\
\hline $\begin{array}{l}\text { Point-locations are inherent to } \\
\text { the line they are located on }\end{array}$ & $\begin{array}{l}\text { Members exist independently } \\
\text { of the sets they are in }\end{array}$ & $\begin{array}{l}\text { Numbers exist independently of the } \\
\text { sets they are in }\end{array}$ \\
\hline $\begin{array}{l}\text { Two point-locations are distinct } \\
\text { if they are different locations }\end{array}$ & $\begin{array}{l}\text { Two set members are distinct } \\
\text { if they are different entities }\end{array}$ & $\begin{array}{l}\text { Two numbers are distinct if there is } \\
\text { a nonzero difference between them }\end{array}$ \\
\hline Properties of the line & $\begin{array}{l}\text { Relations among members of } \\
\text { the set }\end{array}$ & Relations among numbers \\
\hline A point $\mathrm{O}$ & An element $\ll 0 »$ & Zero \\
\hline A point $\mathrm{I}$ to the right of $\mathrm{O}$ & An element « $1 »$ & One \\
\hline Point $\mathrm{P}$ is to the right of point $\mathrm{Q}$ & A relation " $\mathrm{P}>\mathrm{Q}$ " & $\begin{array}{l}\text { Number } P \text { is greater than number } \\
Q\end{array}$ \\
\hline Points to the left of $O$ & $\begin{array}{l}\text { The subset of elements } x \text {, with } \\
0>x\end{array}$ & Negative numbers \\
\hline The distance between $\mathrm{O}$ and $\mathrm{P}$ & $\begin{array}{l}\text { A function } d \text { that maps }(\mathrm{O}, \mathrm{P}) \\
\text { onto an element } \mathrm{x} \text {, with } \mathrm{x}>0\end{array}$ & The absolute value of number $\mathrm{P}$ \\
\hline
\end{tabular}

Neural links tend to survive if the co-activation of their ends is repeated regularly (that is, there are relatively few situations where the origin node is "on" and the target node is "off"). If a mapping between two domains is not inference preserving, the co-activation of inferences will not be very regular, and the links between them will weaken. ${ }^{1}$ Therefore, those neural mappings that survive and become dominant are inference preserving. This is the criterion of best-fit: only the most inferentially conservative metaphors survive.

1 Here is a sketch of an explanation: suppose, that $A$ and $B$ in one domain are connected by an inference ( $A$ activates $B$ ), and that there are links from $A$ to $C$ and from $B$ to $D$, where $C$ and $D$ lie in another domain. Then, an activation of A will activate $C, B$, and in turn $D$. But if $D$ is not an inference from $\mathrm{C}$ (does not represent a situation or action that follows from $\mathrm{C}$ ), the former will often be inhibited in such situations. Due to this inhibition, in many situations $\mathrm{B}$ will be activated and $\mathrm{D}$ will not, so the link from B to D may break. Therefore, if there is no inference link from C to D, the link from B to D may disappear. 
Since no two domains are perfectly isomorphic to each other, metaphorical linking may involve only some nodes of the domain or target, or may enforce the creation of new nodes or inferences in the target domain that will fortify the inference preserving performance of the mapping. This is a form of abstraction, which includes the selection of only some aspects of a domain or the formation of new entities and conclusions. Nevertheless, neural links can sustain some degree of occasional inhibition, so some level of mismatch between the inferences in the two domains is tolerable.

The overall picture suggested by the theory is that mathematical reasoning is implemented by a system that is in some way reminiscent of formal mathematical languages: we have nodes that play the role of propositions, neural links that play the role of inferences, and mappings that are somewhat like morphisms. But this analogy should not be pursued too far, as it fails to distinguish between a genuinely "natural" metaphor, grounded in intuitive and widespread embodied practices, and a forced formal metaphor, which is an ad-hoc rhetorical tool for explaining specific points.

Indeed, according to the modular story, some aspects of contemporary formal mathematics depend on "unnatural" ad-hoc metaphors (such as thinking of a continuous line as a set of discrete coordinates), which are in conflict with our natural understanding (in this case, of the difference between continuity and discreteness). Such metaphors violate some of our inferences, and lead to gaps between our intuition and formal mathematical theory, rendering mathematics less accessible and more artificial.

\section{THE DYNAMIC STORY}

The dynamic story presented here starts from Freeman's research on smell perception in animals. If one follows popular presentations of brain science, one might expect each different smell to be represented by a dedicated neuron or small circuit in the brain. But this is not what Freeman found. He acknowledges that each smell activates specific kinds of chemical sensors in the nose. But since there are many sensors all over the nose, different sensors are activated for different sniffs of the same smell. The pulses from these different sensors should integrate to a common representation, if we expect the same smell (stimulus) to generate the same behavior (response). Freeman did find such an integration, but it was not localized to a specific neuron or a small local circuit in the brain. He found that each smell was represented by a large scale typical pattern measured by EEG across the surface of the olfactory bulb.

But the stimulus-response or representation language used above is, according to Freeman, problematic. First, not just any smell gives rise to a regular pattern - only smells associated with a meaningful reinforcement (reward or punishment). Second, different individuals produced different patterns of the same smell. These two facts mean that the processing of smells is selectively formed by the brain itself, and not simply given or hardwired.

Third, the pattern associated with a smell was not activated automatically whenever the smell was introduced. The activation of the smell-specific pattern depended on the general situation of the animal (sleepy, hungry, etc.). Finally, the pattern gradually changed over time - it would continuously transform over a period of several days. Training the animal to recognize and assign meaning to new smells would also change the patterns associated with the old smells. These two facts mean that the brain activity pattern is not a rep- 
resentation of the smell itself, but rather an expression of a combination of the smell, the animal's general situation and the animal's history.

More generally, Freeman explains that the intentions, expectations and actions of an individual feed into sensory cortices, activating or inhibiting components in these areas independently of external stimulus, thus leading to the discovery of a sought real or imagined stimuli. Since sensory cortices receive inputs from the brain itself, the perception areas of the brain do not simply represent external stimuli, but express the integration of such stimuli with intentions, expectations, history, general situation and activity pattern.

The conclusion that Freeman draws is that sensory data processing is a self-organizing dynamical system. Sense organs and other brain areas provide the system with input that sets it in motion, the "trajectory" of the system is determined by the neural links formed so far, and the system's activity tends to converge to "attractors" - regular patterns of activity (or default) associated with known smells (or lack thereof). This dynamic system has unboundedly many different possible "input states", but they tend to converge to a more limited set of attractors (or dissipate back to a default state). It may also be chaotic in the sense that small differences in "input states" may converge to different attractors.

Since the continued learning activity of the individual takes place in a changing environment (both internal changes of a living body and external environmental changes), the dynamic system is exposed to ever changing stimuli, and changes its internal links and structures accordingly. This is why, as time goes by, the attractors that express a given smell in a given context change.

Freeman claimed that this organization of sense perception is mirrored in higher cognitive functions as well, including computation and abstract reasoning. Following on this suggestion, mathematical "stimuli" (representations) would not activate specific nodes in small domains as suggested by modular stories, but would rather be reflected in large scale patterns of activity along larger brain areas. These patterns will be expressions of the mathematician's general situation, intention, expectation and history, together with the mathematical signs or diagrams to which she is exposed.

A mathematical inference may be thought of as an attractor of the dynamic system -a stable pattern of activity that is repeated, given similar stimuli in similar situations. A transfer of reasoning from one context to another, then, will not be instantiated by an inter-domain metaphor, but by converging to the same attractor despite exposure to stimuli from different contexts. For example, given the stimulus of a number added to itself, our cognitive process may converge to an attractor associated with saying that the sum is greater than the initial number. The same attractor on the same brain component may be reached by a very different stimulus, say adding a geometric shape to itself. Here we do not associate reasoning with two separate modules - one about numbers and the other about shapes, each relatively autonomous, and linked to the other by metaphors.

This story opens a much wider gap between our formal mathematical languages and the brain dynamics that underlie mathematical reasoning. We can no longer think of the two as very similar. Instead, we need to think in terms of unstable causal relations between combinations of mathematical representations, expectations, intentions, actions and history on the one hand, and mathematical performance on the other. 
Four aspects of mathematics, and how the modular and dynamic stories account for them

Here I will consider four aspects of mathematical practice: consensus, generality, innovation and reference. I will try to describe them from the point of view of our two stories. The purpose of this exercise is not to explore the explanatory power of each story, but understand the pictures of mathematics that each story paints around the phenomena. I only point out the explanatory limitations of the theories where this entails complementing the resulting image of mathematics by some non-cognitive stories.

\section{Consensus}

One of the most striking phenomena about mathematics is the strong consensus among experts. Mathematicians tend to agree on the validity of mathematical arguments much more consistently than other scholars do on the validity of their respective arguments. This does not mean that there are no disagreements among mathematicians concerning the validity of arguments. It only means that given enough motivation, time and opportunity for communication, the mathematicians are far more likely to reach agreement concerning the validity of a disputed argument (or extract more refined interpretations, each of which is consensually valid or not) than scholars in other fields.

This does not mean that mathematicians are more likely to agree on the importance, beauty, originality, difficulty, interest or meaning of arguments. Moreover, this does not mean that mathematicians can resolve all doubts concerning the truth (in the restricted sense of provability or any other sense) of mathematical statements. Indeed, if an argument that is meant to prove a given statement is presented, mathematicians may agree that the argument is invalid or incomplete without being able to deny the argument's supposed conclusion (the fact that this proof fails does not mean that subsequent proofs will also fail).

Consensus over validity is not always guaranteed, of course. Cauchy's original proof of the continuity of the limit of a convergent series of continuous functions is typically rejected as invalid, but some commentators argue that this rejection depends on a misunderstanding of Cauchy's notion of continuity, functions and numbers (for a review see Schubring 2005, 431-436). A more recent disagreement can be found in the following quotation: "It had been anticipated that O. Gabber would be a co-author of the present paper. He preferred to withdraw, so as not to be co-responsible for the errors and inaccuracies in it. He is no less responsible for many of the ideas that we exploit...” (Beilinson et al. 1982, 7). Apparently, the authors could not agree at the time on whether all theorems were stated and proved correctly.

But such statements are exceptions, not the rule. Even in the case of a complicated, long and deep endeavor such as Wiles' proof of Fermat's last theorem, only two years (1993-1995) passed from the moment of announcement, through the phase of review and finding an error, to correcting the error and a more or less consensual endorsement. In physics such a paper might trigger decades of research programs before it would become a more or less consensual working theory. In the social sciences it might be the hip new theory that attracts a lot of critique and debate. But in mathematics, the proof reached consensual endorsement within a couple of years.

How would the different cognitive approaches account for this consensus? It is clear that the modular story is much more amenable to consensus. The modular story begins with more or less universal, everyday embodied actions shaped by our common evolution- 
ary history. This obviously lays the ground for consensus. Furthermore, mathematical metaphors are constrained by the "best fit" criterion for inference preservation. Out of the various ways to construct mappings between mathematical domains, only those that maximize the preservation of inference survive naturally. This further eliminates variety in mathematical reasoning.

However, there is a contingent element in the construction of mathematical metaphors. According to the theory, mathematical metaphors emerge between those domains that tend to be activated together. This tendency for co-activation may be a cultural-historical variant, rather than a universal. This may explain why the classical Greek mathematicians, who strictly segregated arithmetic and geometry, accompanied diagram 1 below with the theorem "If a straight line is cut at random, then the square on the whole equals the sum of the squares on the segments plus twice the rectangle contained by the segments" (Euclid's Elements II.4), whereas we are more likely to think of the diagram as an expression of the algebraic identity $(a+b)^{2}=a^{2}+b^{2}+2 a b$ (in diagram 1, the left hand side is the entire divided square, the first two terms on the right hand side are the large and small subsquares respectively, and the third term on the right refers to the two rectangles). The metaphor relating the geometric situation to an arithmetic/algebraic formula failed to emerge (or was inhibited) in the cognition of classical Greek geometers. ${ }^{2}$

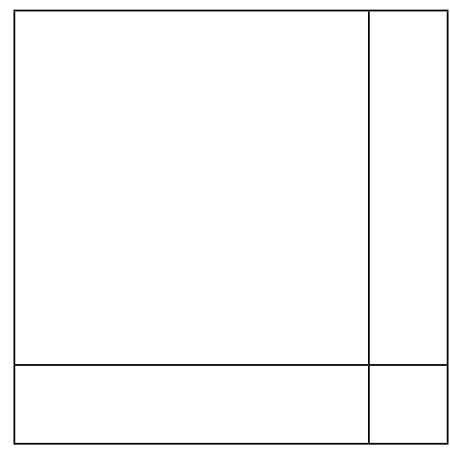

Diagram 1

Euclid's Elements II.4

There are other sources for mathematical variety, namely the contingencies of imposing ad-hoc "unnatural" mathematical metaphors (Lakoff and Núñez (2000) criticize, for example, our mainstream arithmetized and formalized mathematical metaphors for continuity, infinitesimals and limits of sequences of curves, and suggest alternatives). But the rigid cognitive infrastructure behind successful mathematics provides a good basis for a consensual practice.

The dynamic story faces a much bigger challenge here. How can a somewhat chaotic, self-organizing dynamical system, that takes into account individual situation, action, in-

2 A full explanation in terms of the modular story would of course have to reconstruct the entire relevant systems of metaphors and point out the differences that led Greek and modern mathematics in different directions. 
tention, expectation and history, produce the same result when instantiated by so many different individual embodied minds? The only way out is to establish that mathematics is not as consensual as it seems.

Indeed, on some levels, mathematical validity is not very consensual. Mathematical practice is full of errors, misunderstandings and disagreements of the kind anticipated by a dynamic model. The fact that mathematicians can reach consensus (provided they have enough time, motivation and opportunity to communicate) may be explained by something inherent to the mathematical process of communication, rather than to mathematical cognition itself.

This approach is supported by a historical outlook. The Greeks, for example, had a more or less strong consensus around the basic mathematics captured in Euclid's Elements, but beyond that, Greek mathematics was a rather polemical scene (Netz 2004, 62; Lloyd 2012). By Arabic times, the accumulation of challenges raised against Euclid's Elements required an answer in the form of Ibn al-Haytham's Solution of the doubts concerning Euclid's book (Ighbariah and Wagner, 2018). The infamous sixteenth-century dispute between Tartaglia and Cardano/Ferrari is known as a debate over precedence and publication rights, but in fact included disputes about the very correctness of proposed solutions (Bortolotti 1933).

This polemical style of mathematical disputation, quite common at the time, might explain why juridical rhetorical structures were imported into mathematics from legal discourse at the beginning of modernity (Cifoletti 1992, 1995). But juridical rhetoric did not resolve the insistent debates over the legitimacy of infinitesimals that extended from early modernity well into the $19^{\text {th }}$ century (e.g. Alexander 2014, Carnot 1813). Negative and complex numbers suffered a similar fate (Schubring 2005). Disputes that superposed mathematical validity, social cliques and generation gaps remained popular in the $19^{\text {th }}$ century (Ehrhardt 2010, 2011; Wagner 2014, 2016). The Italian school of algebraic geometry was confronted with enduring disputes over mathematical validity as late as the $1930 \mathrm{~s}$ (Brigaglia and Ciliberto 2004).

Mathematics has only become as consensual as we expect it to be today during the last century or so, but it did have (somewhat weaker) means to reduce dissensus in the past as well. Examples are the "formulaic" (but not formal!) language of elementary Greek geometry (Netz 1999), empirical controls during the time when mathematics was more closely associated to natural sciences, and Eulerian over-determination of methods (namely, when different suspect arguments led to the same result, it was more likely to be endorsed, see Sandifer 2007, ch. 31).

Today the most obvious means to guarantee consensus is semi-formalization. Where authority, experience, intuition and standard mathematical toolboxes fail to resolve a mathematical dissent, partial translations that increasingly approximate some kind of formal-axiomatic system can serve as decisive arbitrators. ${ }^{3}$ Those arguments and problems that we believe to be non-translatable to formal languages, or that remain undecidable given such

3 I am referring here to the kind of everyday dispute between mathematicians that remains undecided until one side "writes it down in more detail" - that is, according to the standards of a more rigorous level of semi-formalization, where many such levels separate a real-life proof from a fully formalized one. Writing down a proof in a purely formal-axiomatic language is, with few exceptions, only something that we believe to be attainable, not something we actually attain (either because it is useless or because it would require an unreasonable amount of time). 
translation, are simply exiled from mathematics to less consensual domains, such as philosophy or natural sciences. Thus, some problems that had been considered mathematical later became non-mathematical (the paradoxes of infinity were exported to philosophy and theology, ${ }^{4}$ and the validity of the parallel postulate became a physical question).

My point is that since the dynamical story does not seem to be able to account for consensus by itself, it would have to relegate consensus to a non-cognitive aspect of mathematics. The obvious candidate, as far as I can see, is the historically situated intersubjective processes of deliberation and disciplinary demarcations based on semi-formalized languages. ${ }^{5}$ However, we do not have to draw a sharp line to distinguish "internal" cognition from "external" formal representation and inter-subjective deliberation. Extended cognition would view different brains, signs and tools as semi-autonomous elements of a single dynamic cognitive system. If one wishes to understand cognition in this way, one links cognition to social authority and power, as signs and tools do not float in a discursive universe of purely free and good will, but are also subject to mechanisms of social control.

\section{Generality}

Mathematical reasoning is famous for its capacity to draw a general result from a particular representation. This applies to geometrical representations, where a diagram of a specific triangle can support a general theorem about all triangles, as well as to algebraic representations, where manipulating the particular variable sign $x$ can provide us with knowledge relevant to all numbers.

The modular account of this phenomenon would rely on the universal structures that cognition extracts from representations. Indeed, when two cognitive domains are linked, they are never linked perfectly, because no two cognitive domains are perfectly isomorphic. The act of linking selects a subset of the two cognitive domains (possibly adding new entities to either domain) so that inference preservation is maximized. When we reason mathematically, that is, when we apply the mappings that link the relevant cognitive domains, we extract from the representations on which we operate a core that is approximately inferentially isomorphic to an abstract and general mathematical situation. As a result, we do not reason on the specific representation, but on its mental abstraction, which guarantees a general result.

There is some interesting literature on procedures that could fit this narrative in the context of diagrams. Shin (1994) shows how we can operate abstractly on Venn diagrams.

\footnotetext{
To the extent that such paradoxes are formalizable, they are still a mathematical concern.

5 One may ask why other sciences cannot apply the same strategy for generating consensus. A general discussion of this point is available in Wagner $(2017$, ch. 3). Briefly put, other sciences are constrained by their empirical references (natural or social sciences) or intimate relation to the ambiguities of human language (cultural studies and lettres in the French academic sense). Therefore, they cannot afford to assign jurisdiction to a formalized arbitration mechanism such as a formal language. Those aspects of science that can be formalized (e.g. game theory, formal logic, asymptotic computational complexity, axiomatic physics) end up being re-categorized as part of mathematics. In a sense, formalization has become so synonymous with mathematics, that no other science can rely on it for consensus without becoming mathematical itself.
} 
Avigad et al. (2009) extend the observations of Manders (2008) to rigorously codify general processes of reasoning implicit in Euclidean geometric diagrams. This process is even more salient in the context of variables and other general symbols, where we often reason formally, following syntactic rules, rather than semantically, following the meaning of signs. Of course, the use of diagrams may be misleading, but the modular story would relate that phenomenon to problematic metaphors.

A dynamic story of cognition would find it hard to explain how we reason universally on particular diagrams. If reasoning is the integration of stimulus, expectations, intentions, general situation and individual history by a somewhat chaotic dynamical system, why would different diagrams lead the dynamic system towards the same attractor (expressed as a practice of reasoning or inference)? Moreover, even if generalizations do occur (in the sense of triggering the same reasoning by different representations), how come the generalization emerging from, say, a bunch of triangle diagrams, corresponds to the logical-mathematical category of triangle, and not to some other ad-hoc, contextually emergent category?

The answer, as above, would be that we don't always reason correctly from diagrams, and that different diagrams can lead us to different results. The extreme cases are diagrams that represent a qualitatively incorrect situation, as in the "proof" that all triangles are isosceles, which is based on drawing a certain intersection of lines inside the triangle rather than outside (Kline 1972, 1006-1007; see diagram 2). What makes this "proof" appear convincing for people with standard school training in Euclidean geometry is probably the familiar diagrams of bisectors intersecting inside a triangle with perpendiculars to the sides from the intersection point. These diagrams are a prominent part of standard Euclidean geometry courses. They make the wrong diagram appear plausible, and trigger the dynamic process of reasoning toward mathematically wrong results. Even algebra provides examples for wrong inferences that are based on over generalizations of certain patterns (considered here as visual patterns, a sort of diagram made of symbols). This is the case of " $x^{2}=x \mathrm{im}$ plies $x=1$ ", which may appear to hold because it is part of our standard tool-box when $x$ is replaced by positive numbers (this kind of trick is key to many $1=0$ "proofs").

My point is that reasoning generally from specific diagrams in mathematical practice cannot be reduced to some cognitive mechanism that would extract correct generalizations from particular representations. The underlying cognitive mechanism should actually account for the widespread errors due to diagrams that we find in actual practice. We should also note that qualitatively correct diagrams may also mislead, when they represent only one of several possible cases. Moreover, "incorrect" diagrams are not only an obstacle; they can also serve to advance creative reasoning (e.g. Grosholz (2007) and Wagner (2010), where the infinite is depicted as finite and a certain unknown value is wrongly depicted as 1 , respectively). This generally occurs when diagrams express several incompatible but relevant perspectives at once. ${ }^{6}$ This holds for algebraic representations as well: different representations can suggest different inferences, and the ambiguity of algebraic notations can be highly productive (Wagner 2017, ch. 4; 2009; Arcavi 1994).

6 Ambiguity can be productive, of course, even if it's not contradictory. See Giardino (2013) for a discussion. 


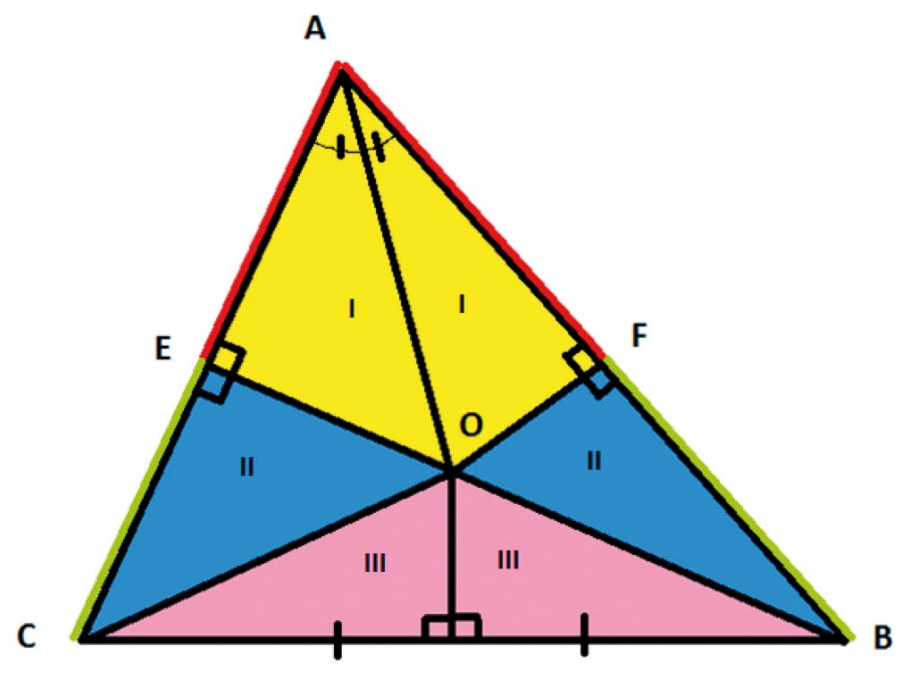

Diagram 2

"Proof" that every triangle $A B C$ is isosceles: "one constructs the angle bisector at $A$ of triangle $A B C$ and the perpendicular bisector of side $B C$ [as in the figure]. If these two lines are parallel, the angle bisector is perpendicular to $B C$ and the triangle is isosceles. We suppose, then, that the lines meet at $O$, say, and we shall still "show" that the triangle is isosceles. We now draw the perpendiculars $O F$ to $A B$ and $O E$ to $A C$. Then the triangles marked I are congruent, and $O F=O E$. The triangles marked III are also congruent, and $O B=O C$. Consequently the triangles marked II are congruent, and $F B=E C$.

From the triangles marked I we have $A F=A E$. Then $A B=A C$ and the triangle is isosceles"

(Kline 1972, 1006-1007)

All the above fits well with the dynamic story. Indeed, if one follows this story, a diagram is a trigger for the convergence of the system to certain attractor (here, some inference or other expression of reasoning). Each attractor has a basin of attraction, namely, those situations and stimuli (here, diagrams) that trigger convergence to the attractor. The scope of such a basin of attraction needn't be identical with any mathematical category: it could include only some of the mathematically relevant diagrams (those we are most used to associate with that form of reasoning), and may even include some diagrams that are mathematically incorrect, leading to mathematically false or mathematically new and creative inferences. These operational, relative and constantly changing boundaries of the basin of attraction of some act of reasoning would escape the problems inherent in attempts to understand reasoning in terms of extraction of an essence or definitional features from particulars.

But the problem remains: given such a dynamic story, how can we reason universally and consensually on different particular representations, as we do, for example, in Euclidean geometry? A dynamic account cannot reduce this to mathematical cognition alone. Correct general reasoning from particular representations would require training with many different alternative representations and the accompaniment of other cognitive supports, such as textual or verbal commentary. As in the case of consensus, if we follow the dynamical story, mathematical generalization from specific representations would have to be based on inter-subjective controls, and not only on individual cognition. 


\section{INNOVATION}

Many believe that mathematics envelops all its truths and entities in its definitions and axioms. But even those people who believe that these definitions and axioms are also somehow enveloped in our most basic interactions with numbers and figures, won't be able to deny that there's a history of discovery in mathematics, and therefore innovation. Alternatively, one may consider mathematics to involve a strongly conventional or constructive element, and therefore proper invention of new mathematical entities. The question for us, however, is: what is the image of mathematical innovation supported by the modular and dynamic cognitive stories?

The modular approach relies on co-activation of domains to initiate new mathematical metaphors. This means that if some new cultural, technological or other circumstances tend to make simultaneous use of two previously dissociated domains, some new mathematics may result. For example, if arithmetic and geometric practices apply in distinct situations, but, for some reason, at a certain time and place, begin to be practiced in close proximity to each other (as different approaches to the same problem, or in a specific practical context that uses both intermittently), then metaphors may link the two domains, and possibly carry new entities with them.

More specifically, geometrical magnitudes that did not have a numerical expression, may give rise to new numbers, following "a geometric magnitude is a number" mapping. A classical example is the introduction of irrational roots. Consider a right angle triangle

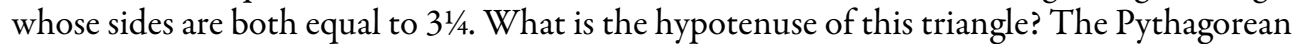
theorem is supposed to provide an answer. But the sum of the squares of the sides is $169 / 8$, and this number has no rational root, and so, in a system where "number" is synonymous with a positive integer or a fraction of positive integers, the hypotenuse will not have a numerical value. When this very problem appears in Bhāskara II's Līlāvatī (12 ${ }^{\text {th }}$ century India, but this maneuver is echoed in earlier texts, and in the Arabic and medieval European cultures as well), the internal commentary reads: "Since it [169/8] has no root, the hypotenuse is this very [number] at the root [sign]". ' In other words, lacking a precise numerical root, this magnitude is to be expressed by the juxtaposition of the root sign and the number whose root is sought, yielding (in modern notation) $\sqrt{169 / 8}$. As a geometrical magnitude is carried into the world of number, a new kind of number is born.

Another example, central to the work of Lakoff and Núñez $(2000,233-235)$ is the introduction of infinities and infinitesimals. Consider the unbounded, unending sequence of positive integers. Project onto this arithmetical domain the inferences of the domain of processes that reach an actual end. This imposes a new final number, which follows all natural numbers, called infinity.

These examples of innovation all depend on transferring something from one domain to another, thereby creating a new entity. But if we replace the modular approach by a dynamic one, it would make little sense to divide mathematics into components which exchange inferences or entities. In a dynamic setting, if a new geometric representation is provided, and I intend or expect to do an arithmetical operation, the dynamical system might converge to an existing attractor (transferring an inference from geometry to arithmetic), or something completely different may happen (which may be either nonsense or a new idea).

Asya mūlābhāvāt karaṇigata evāyaṃ karṇaḥ (Sarma 1975, 276, quoting the prose following verse 139 of the Lìlāvatīi). 
So in a self-organizing dynamic setting, we can expect not only the transfer of inferences from one practical context to another, but also emergence of new phenomena, that are not reducible to previous practices. One example for such emergence, which goes beyond transfer from context to context, is the emergence of Arabic algebra. This $9^{\text {th }}$ century innovation, which kept evolving over several centuries, builds on methods for solving linear equations in one or more unknowns, combined with the Babylonian methods for finding the sides of a rectangle, given some combinations of its area, diagonal and sides (Oaks and Alkhateeb 2005, 416-418). But when these methods were consolidated together by Arabic mathematicians, the product was much more than the sum of its parts. The entity that stands out in this new development is the equation (although not yet the symbolic equation of the late Renaissance and early modernity). This is a mathematical unit, which results from arithmetic operations applied to numbers and unknowns, has its own distinctive formal characteristics (expressed by a dedicated language - an artificial language of sorts), and can be subjected to distinct operations. It is the focus of a new set of mathematical practices.

The interpretation of an equation can be geometric or arithmetic, theoretical or practical, so the equation serves as a pivot between these contexts (Oaks 2007, 2009). Equations subsume their different possible interpretations under their unified language. But equations are not a geometric entity brought into arithmetic or vice versa, nor are they metaphorical projections of some elementary embodied practice onto other mathematical domains. They emerge at the meeting point of domains, contexts and terminologies, but are not reducible to a unidirectional hierarchical transfer between domains.

The algebraic language served not only as an analytic tool for solving problems, but also as an organizational principle allowing to catalogue problems and produce complete compendia of solutions (such as Omar Khayyam's list of cubic equations, which were solved geometrically - see Netz 2004). A comparison of earlier treatments of the very problems later solved by Arabic algebraic methods demonstrates this point. None of the earlier systems produced a unified whole encompassing a scope similar to that of Arabic algebra. ${ }^{8}$

All the above aspects are within the scope of the dynamic story. The modular story would be hard pressed to include them, and would therefore require some sort of complementing discursive account of mathematics to account for emergence and organization of knowledge beyond best-fit metaphorical transfer.

\section{REFERENCE}

One of the most widespread philosophical debates concerning mathematics is the ontological debate: what do mathematical signs represent or refer to? The idealization of mathematics goes hand in hand with a platonic stance: mathematical signs refer to eternal ideal objects (or relational structures) whose existence is a-priori and independent of the existence of humans. A more contemporary approach (the indispensability argument) is to claim that due to the inextricable entanglement of mathematics and natural sciences, math-

8 Even systems that are as complex and sophisticated as Arabic algebra, such as Indian algebra, have a clearly different character. Indeed, Indian determinate quadratic algebra is organized less around equations in one variable and more around Babylonian-like problems (e.g. given the product and sum, find the two unknowns). As a result, the two algebras end up evolving in rather different directions - even before we bring in the letter-variable notations of Viète and Descartes. 
ematical signs involved in successful scientific practice should be considered as having a reference no less real than that of the signs representing electrons or large physical bodies - even though the referents of mathematical signs cannot be located in space and time.

Those for whom mathematics is an artificial, mechanical and inhuman science would tend to prefer formalist approaches, reducing mathematics to arbitrary signs and conventional rules. In between these two extremes, there are several other possibilities, including various conceptualist approaches (claiming that mathematical signs refer to ideas or abstractions inherent to the human mind) and modal approaches (according to which mathematical signs refer to possible objects or relational structures).

All approaches confront the same underlying problems. Ontologies that detach mathematics from empirical experience make it difficult to explain its success in describing natural phenomena. Ontologies that detach mathematics from the human mind or experience make it difficult to explain how we come to know mathematics. And ontologies that detach mathematics form ideal eternal forms make it difficult to explain why mathematics is (or appears to be) a-priori and universal. Embodied cognition puts an interesting twist on the ontological story. It obviously grounds mathematics in embodied experience and the embodied mind, but the modular and dynamic approaches take different directions from there.

According to the modular story, a mathematical entity is a mental representation whose inferential structure depends on metaphorical links grounded in embodied experience. A mathematical sign activates the various linked domains involved in the metaphors that represent the relevant mathematical situation, and in turn activates the associated inferences. Since this chain of metaphors is grounded in embodied action, the use of mathematical signs for communication or thinking links back to the body, and, if not inhibited, triggers embodied action (such as movement of the body).

Marghetis and Núñez (2013) record such bodily action among mathematicians, expressing mathematical relations and concepts by corresponding gestures. This holds for pedagogical contexts as well, where children accompany their mathematical learning with gestures (Radford et al. 2007). This also fits with Barsalou's (2009) argument that cognition is implemented by (possibly unconscious) simulations of sensory-motor activity by relevant neuronal populations. One might also interpret mathematical reference as the correlation of different activations of some sensory-motor brain module with a specific activation (a sign) of the same or another module (sign is to be understood here in a concrete sensory-motor sense, rather than a computational or abstract sense).

This story is very different from the one told by the dynamic approach. From the dynamic point of view, mathematical signs do not need a corresponding representation in the brain (some node that is activated as one is exposed to the sign). Instead, they are supposed to bias the brain's dynamical system toward certain attractors (Freeman 2009), which process is not necessarily reducible to the activation of some brain node.

The complex and under-determined relation between a sign and the inferences it triggers is expressed in one of my favorite quotes from the entire history of mathematics: "if 4 were the half of 12 , what would be the $1 / 3$ of 15 " (della Francesca 1970, 48), which has variants in several medieval Iberian and Italian arithmetic treatises. This strikes us as nonsensical. Indeed, 4 is simply not the half of 12 ! But if we consider the context, we quickly realize that this is a question about proportions or the rule of three (suppose 4 kilos of tomatoes cost half of 12 Euros, how many kilos for $1 / 3$ of 15 Euro?). The point is that the language of the problem highlights the tension between the face value of a sign and its corresponding value. In 
this question, the value of the sign " 4 " is 6 , and we are asked for the sign corresponding to the value 5 .

When it comes to more advanced mathematical signs like the variable $x$, ambiguity is not just about the many possible values that it can subsume, but also about its possible undecided fluctuation between several formal-syntactical roles (numerical variable, formal variable, variable over different mathematical domains), as analyzed in Wagner (2017, ch. 4; 2009).

Things get even more intricate when we consider representations of impossible mathematical objects - representations that correspond or refer to no mathematical object that fits the relevant mathematical framework. For example, in order to prove theorem III.10 of Euclid's elements ("A circle does not cut a circle at more than two points", see diagram 3), one draws a diagram of the impossible situation where two circles intersect at four points, and reasons by contradiction. Similarly, in order to find the real solution 4 of the real equation $x^{3}=15 x+4$, Bombelli went through what he himself considered as the "sophistic" (and many others as contradictory or impossible) number $\sqrt{-121}$ (Wagner 2017, 50-51; the fact that imaginary numbers were later successfully incorporated into mainstream mathematics is irrelevant for the cognitive account of those who did not consider them as legitimate mathematical entities).

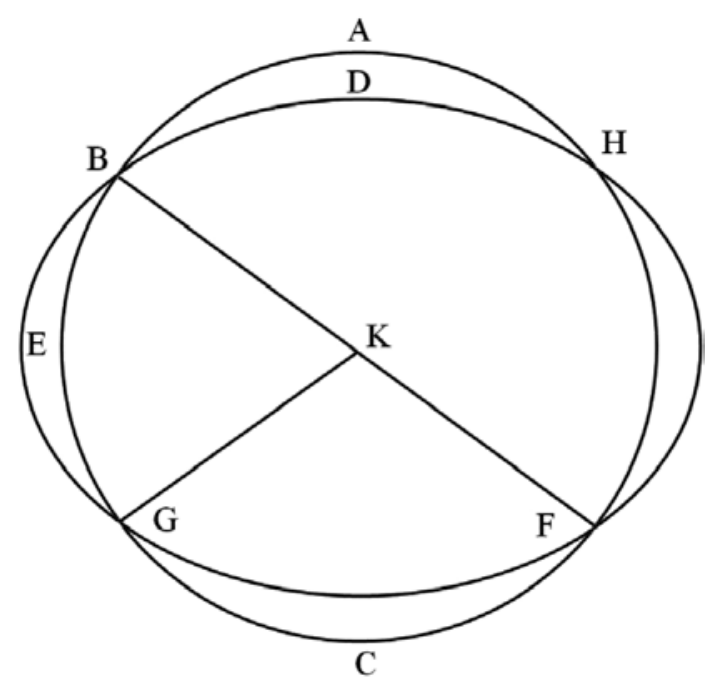

Diagram 3

Euclid's Elements III.10

From a modular point of view this poses difficulties. A sign or a diagram is supposed to activate a practical embodied domain (such as, in the above examples, drawing shapes or taking the side of a square), and relate it to other domains by inference preserving mappings. Representations of impossible objects clearly violate inference preservation between the sensory input and our mathematical concepts. So how come we reason successfully with such representations? In the case of the imaginary numbers, success may be interpreted as the 
slow and hesitant birth of a new mathematical metaphor and entities (the modern complex numbers), but in the case of the counterfactual diagram this account would fail.

The dynamic approach to cognition faces no such difficulties. The diagram or sign of counterfactual or impossible objects need not represent anything. They simply need to bias the dynamical system in favor of certain attractors, that is, the sort of coordination of action, expectation and intention that we term "inference". A reference is not always required. When someone throws a crazy diagram or sign at you, you don't ask what it refers to - you simply catch it.

\section{Conclusion}

The modular story supports consensus and generality without making them as rigid as a formalist account of mathematics would. It allows innovation in the form of carrying inferences and entities from one mathematical domain to another, but is limited in its ability to account for more intricate forms of emergence. It grounds signs in embodiment rather than in external references, but would have trouble accounting for the use of representations of impossible objects.

The dynamic story is difficult to reconcile with consensus and generality, unless one assigns them (plausibly, I believe) to intersubjective communication and formalization, rather than anchor them in individual mathematical reasoning. It allows for the emergence of mathematical innovations that go beyond the transfer of knowledge from one domain to another. Instead of reference, mathematical representations (consistent or absurd) are triggers that direct the dynamic system toward existing or new attractors, giving rise to acts of inference.

Both accounts are fallibilist, but the modular story is perhaps more conservative. It attempts to universalize and rigidify mathematics by grounding it in a robust hierarchy of precise, universal and "natural" metaphors. Divergence is possible, but the criterion of "best fit" (maximal preservation of inferences) marks some mathematical ideas as better or more natural than others.

The dynamic story is even more fallibilist. It makes individual variation, creativity and error a more constituent part of mathematical practice. It also assigns more responsibility to social structures for normalizing mathematics and making it appear absolute. But both stories make mathematics a human creation, rather than an absolute given.

My philosophical inclinations obviously favor the dynamic story, despite its flaws as a scientific theory (e.g., what kind of predictions can one make about mathematical practice based on this story? Without strong neural grounding, which it does not yet have outside sensory cortices, it is almost unfalsifiable and therefore not very scientific - but the modular account does not fare much better on this front). However both approaches, as well as their various theoretical combinations and emendations, are better, I believe, than the absolutist accounts, which force mathematics on us from the outside.

I believe that the cognitive approaches can breathe new life and plausibility into all sorts of conceptualist and social constructivist accounts by protecting them from deteriorating to unguarded nominalism, where anything goes. By binding mathematics to our biologically and socially constrained, yet plastic brains and bodies, they turn mathematics from a regulative ideal or mechanical constraint into a tool to work with. Thinking about mathematics this way, I believe, is much healthier. 


\section{REFERENCES}

Alexander, Amir. 2014. Infinitesimal: How a dangerous mathematical theory shaped the modern world. New York: Scientific American / FSG Books.

Arcavi, Abraham. 1994. Symbol sense: Informal sense-making in formal mathematics. For the Learning of Mathematics 14/3: 24-35.

Avigad, Jeremy, Edward Dean, and John Mumma. 2009. A formal system for Euclid's Elements. The Review of Symbolic Logic 2/4: 700-68. doi:10.1017/S1755020309990098

Barsalou, Lawrence W. 2009. Simulation, situated conceptualization, and prediction. Philosophical Transactions of the Royal Society B: Biological Sciences 364: 1281-89. doi:10.1098/rstb.2008.0319

Beilinson, A.A., J. Bernstein, and P. Deligne. 1982. Faisceaux Pervers Astérisque 100: 7-171.

Bortolotti, Ettore. 1933. I cartelli di matematica disfida e la personalità psichica et morale di Girolamo Cardano. Imola: P. Galeati.

Brigaglia, Aldo, and Ciro Ciliberto. 2004. Remarks on the relations between the Italian and American schools of algebraic geometry in the first decades of the 20th century. Historia Mathematica 31/3: 310-19. doi:10.1016/j.hm.2003.09.003

Carnot, Lazare. 1813. Réflexions sur la métaphysique du calcul infinitésimal. Paris: Mme. Ve. Courcier.

Cifoletti, Giovanna. 1992. Mathematics and rhetoric. Jacques Peletier, Guillaume Gosselin and the making of the French algebraic tradition. Princeton, NJ: Princeton University Press.

—. 1995. La question de l'algèbre. Mathématiques et rhétorique des hommes de Droit dans la France du xvie siècle." Annales. Histoire, Sciences Sociales 50/6: 1385-1416. doi:10.3406/ahess.1995.279438

Della Francesca, Piero. 1970. Trattato d'abaco. Dal codice ashburnhamiano $280\left(359^{*}-291^{*}\right)$ della Biblioteca Medicea Laurenziana di Firenze. Ed. Gino Arrighi. Pisa: Domus Galilaeana.

Ehrhardt, Caroline. 2010. A social history of the 'Galois Affair' at the Paris Academy of Sciences (1831). Science in Context 23/01: 91-119. doi:10.1017/S0269889709990251

-. 2011. "A quarrel between Joseph Liouville and Guillaume Libri at the French academy of sciences in the middle of the nineteenth century. Historia Mathematica 38/3: 389-414. doi:10.1016/j.hm.2011.02.002

Eriksson, Kimmo. 2012. The nonsense math effect. Judgment and Decision Making 7/6: 746-49.

Ernest, Paul. 2008. Epistemology plus values equals classroom image of mathematics. Philosophy of Mathematics Education Journal 23: 1-12.

Freeman, Walter J. 2000. How brains make up their minds. New York: Columbia University Press.

-. 2009. The neurobiological infrastructure of natural computing: Intentionality. New Mathematics and Natural Computation 05/01: 19-29. doi:10.1142/S1793005709001179

Freeman, Walter J., and Robert Kozma. 2009. Brain neural activity patterns yielding numbers are operators, not representations. Behavioral and Brain Sciences 32: 336-37.

Giardino, Valeria. 2013. A practice-based approach to diagrams. In Amirouche Moktefi and SunJoo Shin, eds. Visual reasoning with diagrams, 135-51. Studies in Universal Logic. Basel: Springer. doi:10.1007/978-3-0348-0600-8_8

Grosholz, Emily R. 2007. Representation and productive ambiguity in mathematics and the sciences. Oxford: Oxford University Press.

Ighbariah, Ahmed and Roy Wagner. 2018. The foundations of mathematics in Ibn al-Haytham writings. HOPOS 8/1: 62-86. doi.org/10.1086/695957

Kline, Morris. 1972. Mathematical thought from ancient to modern times, vol. 3. New York: Oxford University Press.

Lakoff, George. 2008. The neural theory of metaphor. In Raymond W. Gibbs, ed. The Cambridge handbook of metaphor and thought. Cambride: Cambridge University Press. http://dx.doi.org/10.1017/ CBO9780511816802.003

Lakoff, George and Rafael E. Núnez. 2000. Where mathematics comes from: How the embodied mind brings mathematics into being. New York: Basic Books. 
Lloyd, G.E.R. 2012. The pluralism of Greek 'mathematics.' In Karine Chemla, ed. The history of mathematical proof in ancient traditions, 294-310. Cambridge: Cambridge University Press.

Manders, Kenneth. 2008. The Euclidean diagram (1995). In Paolo Mancosu, ed. The philosophy of mathematical practice, 80-133. Oxford: Oxford University Press.

Marghetis, Tyler and Rafael Núñez. 2013. The motion behind the symbols: A vital role for dynamism in the conceptualization of limits and continuity in expert mathematics. Topics in Cognitive Science 5/2: 299316. doi: $10.1111 /$ tops. 12013

Netz, Reviel. 1999. The shaping of deduction in Greek mathematics: A study in cognitive history. Cambridge: Cambridge University Press.

-. 2004. The transformation of mathematics in the early Mediterranean world: From problems to equations. Cambridge: Cambridge University Press.

Oaks, Jeffrey A. 2007. Medieval Arabic algebra as an artificial language. Journal of Indian Philosophy 35/5-6: 543-75. doi: $10.1007 / \mathrm{s} 10781-007-9026-4$

-. 2009. Polynomials and equations in Arabic algebra. Archive for History of Exact Sciences 63/2: 169-203. doi:10.1007/s00407-008-0037-7

Oaks, Jeffrey A. and Haitham M. Alkhateeb. 2005. Māl, enunciations, and the prehistory of Arabic algebra. Historia Mathematica 32/4: 400-425. doi:10.1016/j.hm.2005.03.002

Radford, Luis, Caroline Bardini and Cristina Sabena. 2007. Perceiving the general: The multisemiotic dimension of students' algebraic activity. Journal for Research in Mathematics Education 38/5: 507-530. doi: $10.2307 / 30034963$

Sandifer, C. Edward. 2007. How Euler did it. Washington: MAA.

Sarma, K.V. 1975. Lilavati of Bhaskaracarya with Kriyakramakari. Hoshiarpur: Vishveshvaranand Vedic Research Institute.

Schubring, Gert. 2005. Conflicts between generalization, rigor and intuition. New York: Springer.

Shin, Sun-Joo. 1994. The logical status of diagrams. Cambridge: Cambridge University Press.

Wagner, Roy. 2009. Mathematical variables as indigenous concepts. International Studies in The Philosophy of Science 23/1: 1-18. doi:10.1080/02698590902843351

-. 2010. The geometry of the unknown: Bombelli's algebra linearia. In Albrecht Heeffer and Maarten Van Dyck, eds. Philosophical aspects of symbolic reasoning in early modern mathematics, 229-269. London: College Publications.

-. 2013. A historically and philosophically informed approach to mathematical metaphors. International Studies in the Philosophy of Science 27/2: 109-35. doi:10.1080/02698595.2013.813257

- 2014. Wronski's Infinities. History of Philosophy of Science 4: 26-61.

-. 2016. Wronski's foundations of mathematics. Sci Context 29/3: 241-271.

-. 2017. Making and breaking mathematical sense: Histories and philosophies of mathematical practice. Princeton: Princeton University Press.

Roy WAGNER has a PhD in mathematics (1997) and philosophy (2007). He recently published Making and breaking mathematical sense: histories and philosophies of mathematical practice in Princeton University Press. His fields of interest include history and philosophy of science and critical theory. He is currently chair of History and Philosophy of mathematical Sciences at the ETH, Zurich.

Adress: D-GESS, ETH, Postal Address: 59 Clausiusstrasse RZ-J6, Zurich 8092, Switzerland. Email: roy. wagner@gess.ethz.ch 